Dom. Cien., ISSN: 2477-8818

Vol. 4, núm. 1, enero, 2018, pp. 633-647

Fertilización del plátano con nitrógeno, fósforo y potasio en cultivo establecido

\title{
Fertilización del plátano con nitrógeno, fósforo y potasio en cultivo establecido
}

\section{Fertilization of banana with nitrogen, phosphorus and potassium in established culture}

\section{Fertilização de banana com nitrogênio, fósforo e potássio em cultura estabelecida}

\author{
Jorge S. Vivas-Cedeño ${ }^{\mathrm{I}}$ \\ jvas02@hotmail.es \\ José O. Robles-García ${ }^{\text {II }}$ \\ jorobles55@hotmail.com \\ Ignacio González-Ramírez ${ }^{\text {III }}$ \\ igramirez3gmail.com \\ Diana A. Álava-Cruz IV \\ ing_diana23@yahoo.es \\ Manuel A. Meza-Loor V \\ ing_alexmeza27@hotmail.es
}

Recibido: 20 de octubre de 2017 * Corregido: 20 de noviembre de 2017 * Aceptado: 15 noviembre de 2017

I. Magister en Nutrición Vegetal, Diploma Superior en Educación Universitaria por Competencias, Ingeniero Agrónomo, Docente de la Universidad Laica Eloy Alfaro de Manabí, Jipijapa, Ecuador.

II. Magister en Agroecología y Agricultura Sostenible, Diploma Superior en Educación Universitaria por Competencias, Ingeniero Agrónomo, Docente de la Universidad Laica Eloy Alfaro de Manabí, Jipijapa, Ecuador.

III. Doctor en Investigación en Gestión y Valorización de Recursos en la Colina y los Territorios de Montaña, Docente de la Universidad Laica Eloy Alfaro de Manabí, Jipijapa, Ecuador.

iv. Magister en Docencia y Desarrollo del Currículo, Magister en Gestión de Proyectos, Especialista en Nutrición de Rumiantes, Ingeniera Agropecuaria, Docente de la Universidad Laica Eloy Alfaro de Manabí, Jipijapa, Ecuador.

v. Magister en Gestión de Proyectos, Especialista en Manejo del Cultivo de Palma Aceitera, Ingeniero Agropecuario Mención en Producción Pecuaria, Docente de la Universidad Laica Eloy Alfaro de Manabí, Jipijapa, Ecuador. 


\section{Resumen}

El uso de fertilizantes con recomendaciones y dosis específicas, permiten aprovechar al máximo el potencial productivo del cultivo de plátano barraganete, evitar costos de producción elevados y disminuir el desgaste excesivo del suelo. El objetivo de esta investigación fue determinar el rendimiento y la eficiencia de nutrientes para dosis de nitrógeno, óxido de fósforo y óxido de potasio en cultivo establecido durante el año 2015. El experimento se llevó a cabo en tres localidades del Cantón El Carmen, provincia de Manabí. Estas localidades fueron Sumita Pita (A), Las Palmitas de Agua Sucia (B) y La Y de la Raíz (C). Cada nutriente se aplicó en dosis baja, media y alta, más un testigo sin aplicación de fertilizante. En el caso del nitrógeno, estas dosis fueron 0, 150 y $300 \mathrm{~kg}$ ha1; en el fósforo 0, 60 y $120 \mathrm{~kg}$ ha-1 y en el potasio 0,200 y $400 \mathrm{~kg}$ ha-1. La densidad de cultivo fue de 1700 plantas ha-1. Se pudo determinar diferencias significativas en los rendimientos entre las localidades. E A se obtuvo el rendimiento más alto en dosis de 150-60-200 kg ha-1 de nitrógeno, fósforo y potasio (18 $613 \mathrm{~kg}$ ha-1). En B y C, la dosis de $300 \mathrm{~kg}$ ha-1 de nitrógeno mostró la mayor producción (15 840 y $16687 \mathrm{~kg}$ ha-1 respectivamente). En C, el tratamiento con dosis de $400 \mathrm{~kg}$ ha1 de potasio también presentó alta productividad (16 $893 \mathrm{~kg}$ ha-1). La localidad A alcanzó mejor aprovechamiento en la eficiencia de nutriente.

\section{Abstract}

The use of fertilizers with recommendations and specific doses, make the most of the productive potential of plantain plantain, avoid high production costs and reduce excessive soil wear. The objective of this research was to determine the yield and efficiency of nutrients for nitrogen, phosphorus oxide and potassium oxide in culture established during 2015. The experiment was carried out in three localities of Canton El Carmen, province of Manabí These locations were Sumita Pita (A), Las Palmitas de Agua Sucia (B) and La Y de la Raíz (C). Each nutrient was applied in low, medium and high doses, plus a control without application of fertilizer. In the case of nitrogen, these doses were 0,150 and $300 \mathrm{~kg}$ ha- 1 ; in phosphorus 0,60 and $120 \mathrm{~kg}$ ha- 1 and in potassium 0,200 and $400 \mathrm{~kg}$ ha-1. The culture density was 1,700 plants ha-1. It was possible to determine significant differences in the yields among the localities. The highest yield was obtained at doses of 150-60-200 $\mathrm{kg}$ ha-1 of nitrogen, phosphorus and potassium (18 $613 \mathrm{~kg}$ ha-1). In B and C, the dose of $300 \mathrm{~kg}$ ha-1 of nitrogen showed the highest production (15 840 and $16687 \mathrm{~kg}$ ha-1 respectively). In C, treatment 
with a dose of $400 \mathrm{~kg}$ ha- 1 of potassium also showed high productivity (16 $893 \mathrm{~kg}$ ha-1). Location A achieved better use in nutrient efficiency.

\section{Resumo}

O uso de fertilizantes com recomendações e doses específicas, aproveita ao máximo o potencial produtivo do banana plátano, evita altos custos de produção e reduz o desgaste excessivo do solo. $\mathrm{O}$ objetivo desta pesquisa foi determinar o rendimento e eficiência de nutrientes para nitrogênio, óxido de fósforo e óxido de potássio em cultura estabelecida em 2015. O experimento foi realizado em três localidades de Cantão El Carmen, província de Manabí Estes locais foram Sumita Pita (A), Las Palmitas de Agua Sucia (B) e La Y de la Raíz (C). Cada nutriente foi aplicado em doses baixas, médias e altas, além de um controle sem aplicação de fertilizante. No caso do nitrogênio, essas doses foram 0,150 e $300 \mathrm{~kg}$ ha-1; em fósforo 0, 60 e $120 \mathrm{~kg}$ ha-1 e em potássio 0, 200 e $400 \mathrm{~kg}$ ha- 1 . A densidade da cultura era de 1.700 plantas ha-1. Foi possível determinar diferenças significativas nos rendimentos entre as localidades. O rendimento mais elevado foi obtido em doses de 150-60-200 kg ha-1 de nitrogênio, fósforo e potássio (18 $613 \mathrm{~kg}$ ha-1). Em B e C, a dose de $300 \mathrm{~kg}$ ha-1 de nitrogênio apresentou maior produção (15840 e $16687 \mathrm{~kg}$ ha-1, respectivamente). Em C, o tratamento com uma dose de $400 \mathrm{~kg}$ ha-1 de potássio também apresentou alta produtividade (16 $893 \mathrm{~kg}$ ha-1). A localização A melhorou o uso em eficiência de nutrientes.

\section{Introducción}

El plátano representa uno de los cultivos de mayor importancia a nivel mundial, debido a su crecimiento anual en producción, y a las exportaciones que aumentan del mismo modo, lo cual genera incrementos en las actividades económicas del país (SINAGAP, 2015); sin embargo los rendimientos del cultivo suelen ser bajo, considerando la cantidad de superficie que utiliza y la producción obtenida, Manabí, la provincia de mayor participación en este rubro alcanza 3,06 t ha-1 al año (INEC, 2016).

Para los agricultores, constituye un cul5rrttivo altamente remunerativo por los bajos gastos en que se incurren durante el desarrollo de la plantación y su mantenimiento en relación con otros cultivos.

Sin embargo, durante el año 2015, la producción de plátano en Ecuador disminuyó en 11\% respecto al año 2014. Respecto al mismo año, en el 2015 la superficie nacional cosechada aumentó en un 1\%, mientras el rendimiento experimentó una baja del 12\%. 
El aumento de los rendimientos del cultivo, resulta un problema acuciante en la actualidad si se considera que en el 2015, la importación de plátano aumentó en $10 \%$ respecto al año anterior, lo que significó un aumento considerable de la tendencia en los últimos años en la demanda de este producto (SINAGAP, 2015). El aumento de los rendimientos del cultivo está reconocido por los productores, como uno de los problemas más importantes a solucionar para aumentar sus ganancias.

La productividad del cultivo del plátano es afectada por variados factores que tienen que ver con el medio natural, sobre el cual se ha emplazado el cultivo, con las plagas que afectan al cultivo y con las prácticas de manejo que realizan los productores. En diversos estudios, se ha identificado esta diversidad de razones que afectan sobre todo los rendimientos agrícolas (Parménides, 2014; Combatt et all, 2012; Barrera et all, 2011; Langdom et al., 2008). Se destacan la baja densidad de siembra que permite el desarrollo de condiciones favorables para el desarrollo de enfermedades, la presencia de plantas en diferentes estados vegetativos en la misma plantación, lo que permite el desarrollo y avance de enfermedades como la Sigatoka negra, la deficiente fertilización, tanto por la carencia como por aplicaciones indebidas en tiempo y espacio. Estas y otras prácticas de manejo en muchas ocasiones se deben a la falta de información y capacitación técnica entre los productores.

El uso correcto de los fertilizantes ha contribuido al incremento de los rendimientos de los cultivos y como consecuencia, se han logrado mejoras en la rentabilidad del sistema productivo (Espinosa \& Mite, 2002). Para ello es importante que la fertilización sea acorde a los requerimientos de la planta en su fase vegetativa, cuando desarrolla sus raíces y el pseudotallo (Guerrero, 2011). Debe considerarse además, las condiciones edáficas en la localidad en que se cultiva, debido a que las necesidades del suelo y los rendimientos del cultivo difieren espacialmente (Combatt, Martínez, \& Barrera, 2004) y la eficiencia del nutriente varía considerablemente. A partir de diversos experimentos realizados por Corpoica en diferentes tipos de suelo, Palencia y Gómez \& Martín (2006) manifiestan que "el cultivo de plátano presenta una mayor respuesta agronómica con el uso de nitrógeno, potasio y azufre".

La forma más idónea de medir la respuesta del cultivo de plátano al uso de fertilizantes es determinando la eficiencia del uso de los nutrientes, la cual Stewart, (2007) define como "la manera en que la planta o un sistema de producción utiliza los nutrientes a disposición, para incrementar su rendimiento". A pesar de la poca información que se tiene en fertilización del plátano, las nuevas 
investigaciones están dirigidas a plantaciones jóvenes, sin considerar que en la mayoría de los casos, los productores manejan cultivos en segunda fase o ya establecidos. El objetivo de esta investigación fue evaluar la eficiencia del uso de nutrientes del cultivo de plátano barraganete, mediante la aplicación de diferentes niveles de nitrógeno, fósforo y potasio en el Cantón El Carmen, Provincia de Manabí.

\section{Materiales y métodos}

La investigación se realizó en tres localidades del referido Cantón. El primer ensayo se estableció en Sumita Pita (Sumita) con latitud -0.229620 y longitud -79.463164, a 2 km del centro de El Carmen. El segundo en Las Palmitas de Agua Sucia (Las Palmitas), vía Sumita Pita con latitud -0.229846 y longitud -79.511494, a $3 \mathrm{~km}$ de la ciudad y el tercero, en la localidad de la Y de la Raíz (La Raíz), con latitud -0.302205 y longitud -79.511494 a $7 \mathrm{~km}$ de la vía Venado. Las localidades presentan temperatura promedio de $24^{\circ} \mathrm{C}$ y están ubicadas aproximadamente a $230 \mathrm{msnm}$, con 878.5 horas luz año-1 y precipitación anual de $2800 \mathrm{~mm}$. Los suelos en todos los casos, pertenecen a los Oxisoles, con $\mathrm{pH}$ moderadamente acido.

Se utilizaron plantaciones establecidas, con densidades de 1700 plantas ha-1 aproximadamente. El área total para cada ensayo fue de $2400 \mathrm{~m} 2$, con 408 plantas por localidad. Se dispusieron ocho tratamientos con tres repeticiones, para un total de 24 unidades experimentales de $100 \mathrm{~m} 2$ cada una. Se manejó en un diseño de bloques completamente al azar (DBCA). Los tratamientos consistieron en variaciones de dosis de N-P-K (Tabla 1).

Tabla 1: Caracterización de las dosis de los tratamientos

\begin{tabular}{|c|c|c|c|}
\hline Tratamiento & N (kg ha-1) & P (kg ha-1) & K (kg ha-1) \\
\hline T0 & 0 & 0 & 0 \\
\hline T1 & 0 & 60 & 200 \\
\hline T2 & 150 & 60 & 200 \\
\hline T3 & 300 & 60 & 200 \\
\hline T4 & 150 & 0 & 200 \\
\hline T5 & 150 & 120 & \\
\hline
\end{tabular}


Fertilización del plátano con nitrógeno, fósforo y potasio en cultivo establecido

\begin{tabular}{|c|c|c|c|}
\hline T6 & 150 & 60 & 0 \\
\hline T7 & 150 & 60 & 400 \\
\hline
\end{tabular}

Se aplicaron dosis estandarizadas de nitrógeno, fósforo y potasio 150-60-200 kg ha-1 respectivamente. Uno de los tratamientos (T2), fue con dosis media de todos los nutrientes (150, 60 y $200 \mathrm{~kg} \mathrm{ha}-1 \mathrm{de}$ N-P-K). Para efecto de comparación con el uso de nutrientes se empleó un testigo sin aplicación de nutriente (T0). Las labores de manejo durante el experimento fueron similares para todos los ensayos. El área del experimento se delimitó en terreno plano y semiplano. El control de malezas se ejecutó mediante un control químico con dos litros ha-1 de glifosato y dos controles manuales durante todo el ciclo. El deshoje y eliminación de necrosis en las hojas se realizó cada semana; el deschante o eliminación de hoja seca del pseudotallo, cada dos meses. El deshije fue constante hasta la emisión de la bellota y en ese momento, se seleccionó el hijo con mayor vigor para la sucesión. El control del picudo se realizó mediante trampas con insecticidas incorporados.

La aplicación de nutrientes fue en cuatro etapas, en la primera se aplicó el fósforo al 100\% y en las tres últimas se suministraron el nitrógeno y el potasio fraccionados.

Se midieron las siguientes variables:

- Rendimiento del cultivo en $\mathrm{kg}$ ha-1. Se tomó el peso promedio del racimo $\mathrm{kg}$ ( $\sin$ raquis) de cada unidad experimental, y se multiplicó por el número de plantas ha-1. Los resultados de cada tratamiento fueron comparados con la prueba de Tukey al 5\% de probabilidad.

- Eficiencia de nutrientes. Se utilizaron los parámetros de factor parcial de productividad (FPP) y eficiencia agronómica (EA) en todos los nutrientes. El FPP se calculó dividiendo el rendimiento del cultivo para la dosis aplicada $(\mathrm{RF} / \mathrm{D})$, mientras que la EA se calculó restándole al rendimiento de parcela fertilizada, el rendimiento de parcela sin fertilizar y posteriormente calculando el cociente del resultado, entre la dosis del nutriente $[E A=(R F-R 0) / D]$.

\section{Resultados y discusión}

\section{Rendimientos del cultivo}

En las tres localidades en estudio, los niveles de nitrógeno, fósforo y potasio, influyeron en el rendimiento del plátano en cultivos establecidos. En la localidad de Sumita, el tratamiento con dosis 
estandarizadas (T2) alcanzó rendimientos de $18613 \mathrm{~kg}$, mientras que el tratamiento con alta dosis de fósforo (T5) tuvo rendimientos de $18120 \mathrm{~kg}$. Esta localidad fue la de mayor rendimiento (Tabla 2). En las otras dos localidades se alcanzaron resultados similares. En Las Palmitas, la dosis alta de nitrógeno (T3) alcanzó la producción más elevada con $15840 \mathrm{~kg}$, mientras que en La Raíz el rendimiento más alto fue con dosis alta de nitrógeno y potasio (T3 y T7) kg ha-1 con 16687 y 16893 $\mathrm{kg}$ respectivamente (Tabla 2$)$.

Tabla 2: Rendimiento de la fruta del plátano barraganete kg ha-1 en cultivo establecido con niveles de N, P2O5 y K2O en tres localidades del Cantón El Carmen, Manabí, Ecuador 2016

\begin{tabular}{|c|c|c|c|c|}
\hline \multicolumn{2}{|c|}{ Dosis } & Sumita a & Las Palmitas b & La Raíz b \\
\hline \multicolumn{2}{|c|}{ kg ha-1 } & \multicolumn{3}{|c|}{ rendimiento kg ha-1 } \\
\hline \multirow[t]{2}{*}{$\mathrm{T}$} & $0-0-0$ & $13252,28 \mathrm{~cd}$ & $12054,55 b$ & $12615,13 \mathrm{a}$ \\
\hline & 0 & $14256,82 \mathrm{bcd}$ & $13007,58 \mathrm{ab}$ & $14342,33 \mathrm{ab}$ \\
\hline \multirow[t]{3}{*}{$\mathrm{N}$} & 150 & $18613,07 \mathrm{a}$ & $14475,76 \mathrm{ab}$ & $13290,60 \mathrm{~b}$ \\
\hline & 300 & $14990,91 \mathrm{abcd}$ & $15840,76 a$ & $16687,20 \mathrm{a}$ \\
\hline & 0 & $11629,55 \mathrm{~d}$ & $11616,67 b$ & $13941,13 b$ \\
\hline \multirow[t]{3}{*}{$\mathrm{P} 2 \mathrm{O} 5$} & 60 & $18613,07 \mathrm{a}$ & $14475,76 \mathrm{ab}$ & $13290,60 \mathrm{~b}$ \\
\hline & 120 & $18120,46 a$ & $13265,15 \mathrm{ab}$ & $13783,03 b$ \\
\hline & 0 & $16188,64 a b c$ & $13831,82 \mathrm{ab}$ & $12070,00 \mathrm{~b}$ \\
\hline \multirow[t]{2}{*}{$\mathrm{K} 2 \mathrm{O}$} & 200 & $18613,07 \mathrm{a}$ & $14475,76 \mathrm{ab}$ & $13290,60 \mathrm{~b}$ \\
\hline & 400 & $17270,45 \mathrm{ab}$ & $12801,51 \mathrm{ab}$ & $16893,47 \mathrm{a}$ \\
\hline
\end{tabular}

La relación entre la fertilización y los rendimientos no presenta un comportamiento lineal en todas las localidades (figura 1a). En la localidad de Las Palmitas, bajo el efecto del nitrógeno, los rendimientos son directamente proporcionales al incremento de la dosis, así mismo en el caso del potasio (figura 1c). En la localidad de La Raíz, el plátano presenta un rendimiento más alto en dosis más elevadas de fertilización; este comportamiento confirma lo expuesto por Combatt et al., (2004), cuando asegura que la respuesta del plátano a la fertilización está condicionada por la cantidad de nutrientes en el suelo al inicio de la fertilización. Además de que un balance adecuado del nitrógeno y potasio en la superficie, pueden mejorar el rendimiento del cultivo. Como se aprecia en la influencia delnitrógeno 
Fertilización del plátano con nitrógeno, fósforo y potasio en cultivo establecido

y potasio en la localidad de Sumita, la producción aumenta hasta la dosis media, luego disminuye ante un nivel alto de fertilización.

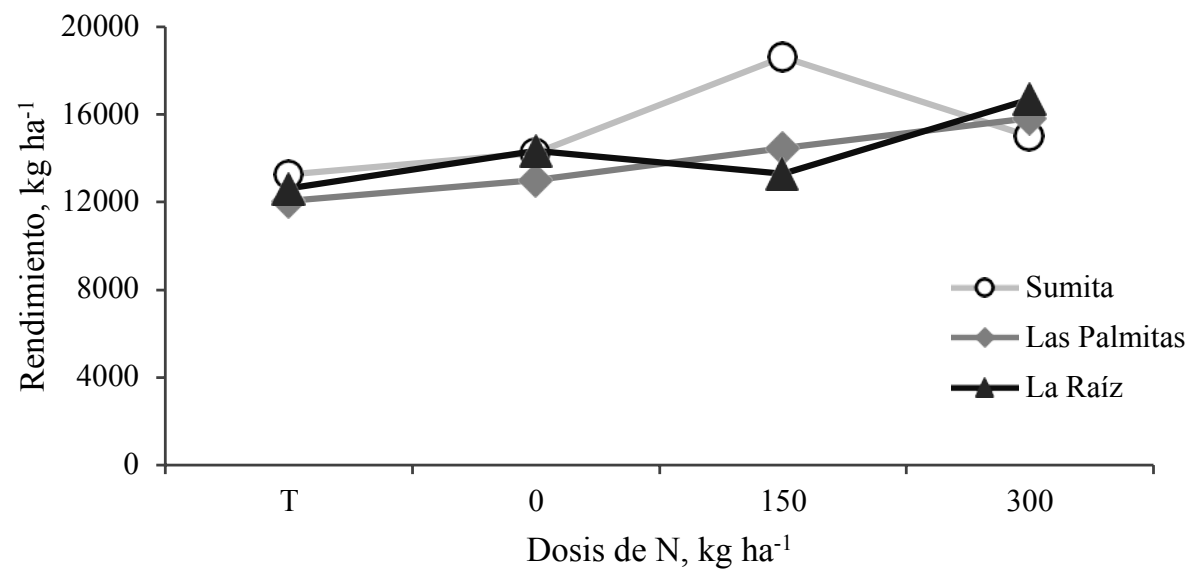

a

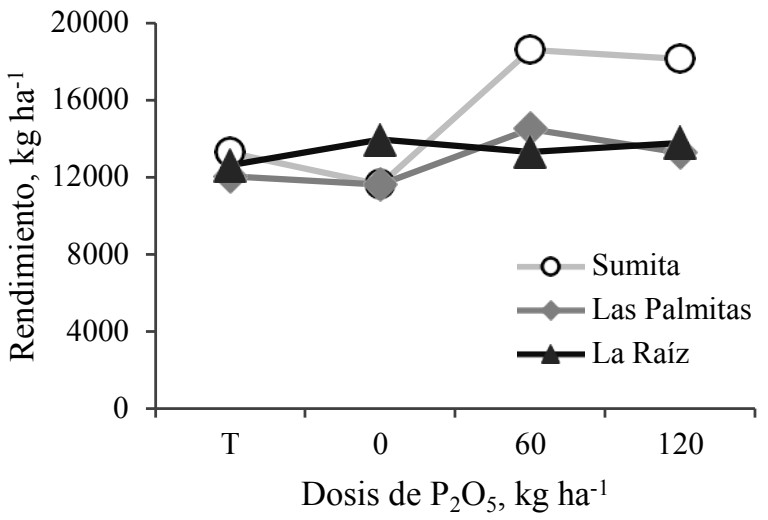




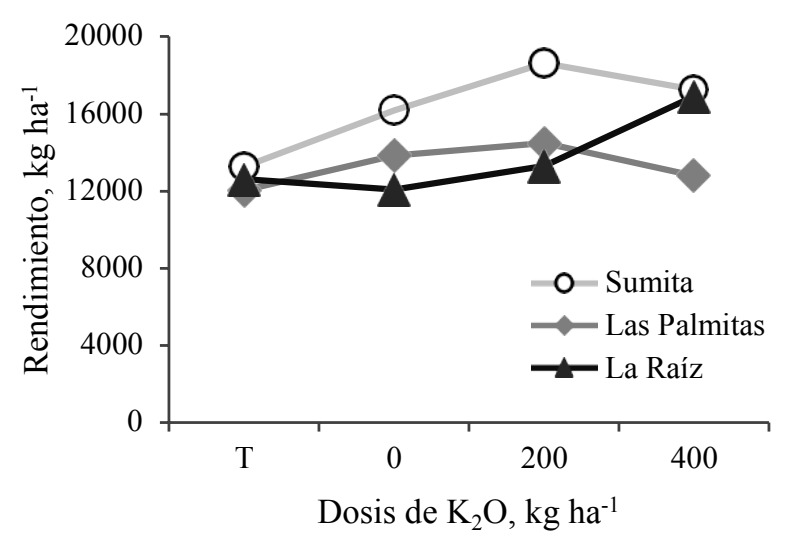

Figura 1. Rendimiento de fruta en cultivo establecido de plátano barraganete, para diferentes dosis de nitrógeno (a), fósforo (b) y potasio (c) en tres localidades del Cantón El Carmen. Provincia de Manabí, Ecuador (2016).

El mayor rendimiento obtenido en esta investigación, es superior al reportado por Furcal \& Barquero (2014), en el curare enano bajo efecto del nitrógeno en dosis de $200 \mathrm{~kg}$ ha-1, ya que alcanzaron una producción de $19720 \mathrm{~kg}$ ha-1. En la fertilización potásica el rendimiento fue similar con la dosis de $200 \mathrm{~kg}$ ha-1 a Furcal \& Barquero (2014), solo que en aquel caso la dosis empleada fue de $375 \mathrm{~kg}$ ha1. Estos resultados son inferiores a los de Combatt et al. (2004), en cultivo de plátano Simmonds, ya que en dosis de $200 \mathrm{~kg}$ ha-1 de nitrógeno y potasio se obtuvo rendimientos de $26656 \mathrm{~kg}$ ha-1.

En cuanto al fósforo (figura 1b), se observa que el efecto de la fertilización con este nutriente no influye directamente en el aumento o disminución de la producción en las tres localidades. Puede inferirse que el fósforo no tiene efecto en el rendimiento de la fruta del plátano. Estos resultados confirman lo expuesto por López (2002), cuando expone que el cultivo de plátano no presenta respuesta en su producción a la fertilización fosforada y las recomendaciones de aplicación, solo son para reposición de los minerales extraídos de la plantación.

\section{Eficiencia Agronómica (EA) y Factor Parcial de Productividad (FPP)}

Entre los objetivos de la Eficiencia de nutrientes, puede destacarse el de aprovechar al máximo la fertilización para incrementar el rendimiento del cultivo por unidad de nutriente aplicado. La mejor manera de evaluar esta variable, es mediante la Eficiencia Agronómica (EA) (Espinosa \& García, 2010). Se calcula mediante la diferencia del rendimiento de la parcela fertilizada y sin fertilizar con la dosis aplicada. Este parámetro es definido como el incremento de producción por unidad de 
nutriente. Para obtener el Factor Parcial de Productividad (FPP), el rendimiento del cultivo se divide entre la dosis aplicada, y se define como producción por unidad de nutriente (Dobermann, 2007). Se considera como la forma más básica para medir la eficiencia del uso de nutriente (Snyder \& Bruulsema, 2007) (Tabla 3).

Tabla 3: Eficiencia Agronómica y Factor Parcial de Productividad del N, P2O5 y K2O del plátano en cultivo establecido en tres localidades del Cantón El Carmen, Manabí 2016

\begin{tabular}{|c|c|c|c|c|c|c|c|}
\hline \multicolumn{2}{|c|}{ Dosis } & \multicolumn{2}{|c|}{ Sumita } & \multicolumn{2}{|c|}{ Las Palmitas } & \multicolumn{2}{|c|}{ La Raíz } \\
\hline kg ha-1 & & EA & FPP & $\overline{E A}$ & FPP & EA & FPP \\
\hline \multirow{2}{*}{$\mathrm{N}$} & 150 & 29 & 124 & 10 & 97 & -7 & 89 \\
\hline & 300 & 2 & 50 & 9 & 53 & 8 & 56 \\
\hline \multirow{2}{*}{$\mathrm{P} 2 \mathrm{O} 5$} & 60 & 116 & 310 & 48 & 241 & -11 & 222 \\
\hline & 120 & 54 & 151 & 14 & 111 & -1 & 115 \\
\hline \multirow{2}{*}{$\mathrm{K} 2 \mathrm{O}$} & 200 & 12 & 93 & 3 & 72 & 6 & 66 \\
\hline & 400 & 3 & 43 & -3 & 32 & 12 & 42 \\
\hline
\end{tabular}

La EA negativa en algunas localidades y para determinadas dosis de nutrientes, obedece a que el rendimiento de algunas parcelas sin fertilizantes superó a las fertilizadas (Tabla 2). En La Raíz, la EAP indica que a mayor uso de nutriente, la producción disminuye a partir de la aplicación. Lo mismo ocurre con la eficiencia de nutrientes del potasio (EAK) con dosis de $400 \mathrm{~kg}$ ha-1 en Las Palmitas, donde el incremento por unidad de nutriente es negativo. Este comportamiento se debe a que la respuesta del rendimiento al uso de fertilizantes, depende del sitio de establecimiento (Espinosa \& Mite, 2008). Además, el fósforo y el potasio, tienen una fácil acumulación en el suelo en formas disponible para las plantas (Fixen, 2010); lo que resulta necesario evaluar es la eficiencia y recuperación de nutrientes a largo plazo (Stewart, 2007).

En la eficiencia del nitrógeno (Figura 2a), la dosis de $150 \mathrm{~kg}$ ha-1 en la localidad de Sumita obtuvo la mayor EA con $29 \mathrm{~kg}$ de incremento en fruta por $\mathrm{kg}$ de nutriente aplicado. El FPP alcanzó $124 \mathrm{~kg}$ de fruta por kg de nitrógeno. La dosis baja de este nutriente (150 kg ha-1) presentó los valores más altos de FPP en todas las localidades. Ello indica que los niveles altos de fertilización, tienen menor eficiencia, tal como lo expresa Boaretto et al (2007); a mayor dosis de nutrientes, el incremento en 
producción se reduce. La EAN en las demás dosis y localidades se mantuvo con valores menores a 10 $\mathrm{kg} \mathrm{kg}$-1. Estos valores son ligeramente inferiores a los obtenido por Furcal \& Barquero, (2014) cuando bajo dosis de 100 kg ha-1 de nitrógeno, alcanzó valores de FPP de 188 y EA de 32 kg kg-1. Confirma entonces lo expuesto por Boaretto et al., (2007). En el experimento de Aristizábal, (2010) con $300 \mathrm{~kg}$ ha-1 de nitrógeno, el FPP (97 kg ha-1) fue mayor al obtenido bajo esa misma dosis, considerando que la variedad evaluada fue el Hartón.

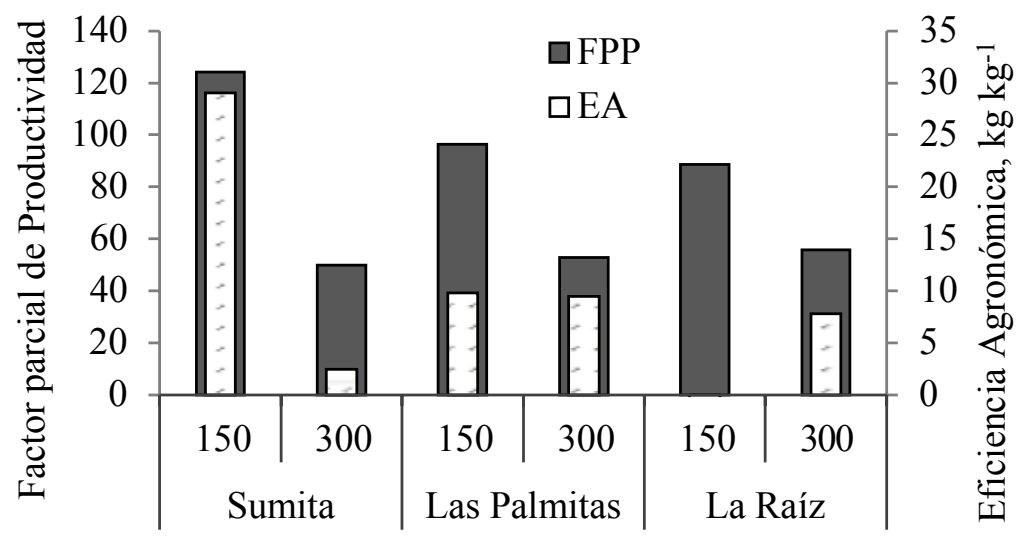

Localidades y dosis de $\mathrm{N}, \mathrm{kg} \mathrm{ha}^{-1}$

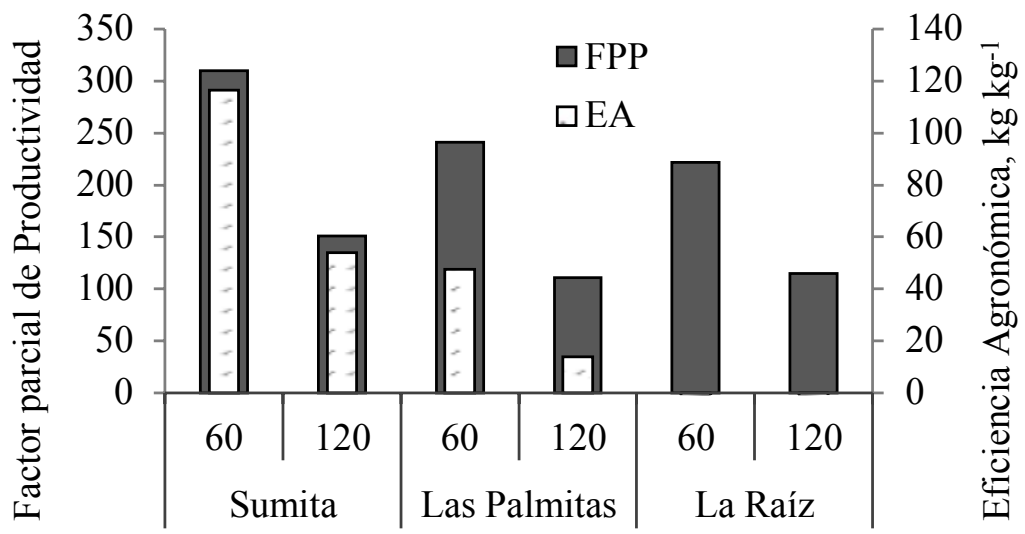

Localidades y dosis de $\mathrm{P}_{2} \mathrm{O}_{5}, \mathrm{~kg} \mathrm{ha}^{-1}$ 


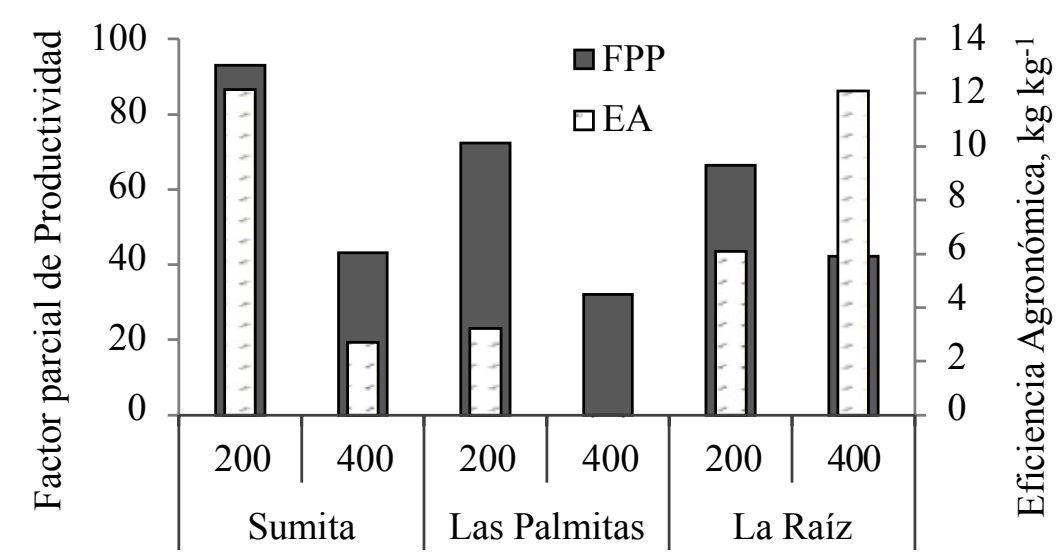

Localidades y dosis de $\mathrm{K}_{2} \mathrm{O}$, $\mathrm{kg} \mathrm{ha}^{-1}$

Figura 2. Factor Parcial de Productividad y Eficiencia Agronómica del N, P2O5 y K2O en un cultivo de plátano barraganete establecido en tres localidades del Cantón El Carmen 2016.

En la localidad de La Raíz, la Eficiencia agronómica de la variación de dosis de fósforo (figura 2b) mostró valores negativos. Ello permite asumir que la fertilización con este nutriente fue perjudicial en la producción al igual que el nitrógeno. En la localidad de Sumita, con dosis baja de fósforo (60 kg ha-1), la EA y el FPP tuvieron los índices más alto con 116 y $310 \mathrm{~kg} \mathrm{~kg}-1$ respectivamente. Estos resultados superaron a los de Furcal \& Barquero, (2013), que en plátano Curare con dosis de $70 \mathrm{~kg}$ ha-1 de P2O5 y 250 de K2O, obtuvieron una EAP de $68 \mathrm{~kg} \mathrm{kg-1,} \mathrm{pero} \mathrm{son} \mathrm{similares} \mathrm{en} \mathrm{el} \mathrm{FPPP,} \mathrm{que}$ llegó a $315 \mathrm{~kg}$ de fruta por $\mathrm{kg}$ de nutriente. La diferencia de resultados en la EA entre localidades puede centrarse en el balance nutricional presente en el suelo (Combatt et al., 2004), cuando existe mayores concentraciones iniciales de nutrientes en los suelos del área de estudio.

El K2O (Figura 2c) mostró resultados de EA menores que el nitrógeno y el fósforo, debido a que el nitrógeno responde a corto plazo en la eficiencia de nutriente, a diferencia del potasio (Stewart, 2007). También el fósforo en dosis más baja, puede obtener mayores niveles de FPP y EA (García \& Salvagiotti, 2015). En la localidad de Sumita, con dosis de $200 \mathrm{~kg}$ ha-1 y en la localidad de La Raíz, con dosis de $400 \mathrm{~kg}$ ha-1, la EAK tiene su potencial productivo con $12 \mathrm{~kg}$ de incremento por $\mathrm{kg}$ de nutriente aplicado. Respecto al FPP el nivel más alto alcanza 97 kg ka-1 con 200 kg ha-1 en la localidad de Sumita. Esta superioridad se repite en las demás localidades. En Furcal \& Barquero, (2014) para la dosis baja de $125 \mathrm{~kg}$ ha-1 de K2O, el FPPK presentó un rendimiento de $146 \mathrm{~kg}$ de fruta por $\mathrm{kg}$ de potasio y una EAK de $8 \mathrm{~kg}$ de incremento de producción por $\mathrm{kg}$ de nutriente. A partir delo 
anterior, los resultados en las localidades de Sumita y La Raíz, expresan mayor eficiencia en el uso del potasio. A pesar de que en estos casos el FPP obtuvo niveles más bajos, la EA permite evaluar de manera ajustada la eficiencia en el uso de los nutrientes en comparación con la parcela sin fertilizar (Espinosa \& Mite, 2008).

\section{Conclusiones}

En el trabajo se evaluó la eficiencia del uso de nutrientes del cultivo de plátano barraganete, mediante la aplicación de diferentes niveles de nitrógeno, fósforo y potasio en el Cantón El Carmen, Provincia de Manabí. Se pudo determinar diferencias significativas en los rendimientos entre las localidades. En A se obtuvo el rendimiento más alto en dosis de 150-60-200 kg ha-1 de nitrógeno, fósforo y potasio (18 613 kg ha-1). En B y C, la dosis de 300 kg ha-1 de nitrógeno mostró la mayor producción (15 840 y $16687 \mathrm{~kg}$ ha-1 respectivamente). En C, el tratamiento con dosis de $400 \mathrm{~kg}$ ha-1 de potasio también presentó alta productividad (16 $893 \mathrm{~kg}$ ha-1). La localidad A alcanzó mejor aprovechamiento en la eficiencia de nutriente.

\section{Referencias bibliográficas}

Aristizábal, M. 2010. Efecto de la frecuencia de fertilización con nitrógeno y potasio sobre el crecimiento, producción y severidad de las Sigatoka del plátano (Musa AAB) Dominico Hartón. Revista Agronómica, 18(1): 19-28

Barrera, J., Combatt, E., Ramírez, Y., 2011. Efecto de abonos orgánicos sobre el crecimiento y producción del plátano Hartón (Musa AAB). Revista Colombiana de Ciencias Hortícolas, 5: 186-194

Boaretto, A., Muraoka, T., \& Trevelin, P. 2007. Uso Eficiente del Nitrógeno de los Fertilizantes Convencionales. Revista Informaciones Agronómicas, 120: 13-14

Combatt-Caballero, E., Novoa-Yánez, R., Barrera-Violeth, J.L., 2012. Caracterización química de macroelementos en suelos cultivados con plátano (Musa AAB Simmonds) en el departamento de Córdoba, Colombia. Acta Agronómica 61(2): 166-176

Combatt, E.; Martínez, G.; y Barrera, J. 2004. Efecto de la interacción de N y K sobre las variables de rendimiento del cultivo de plátano (Musa AAB Simmonds) en San Juan de Urabá, Antioquia. Temas Agrarios 9(1): 5 - 12 
Dobermann, A. 2007. Nutrient use efficiency-measurement and management. Proc. of International Fertilizer Industries Association (IFA) Workshop on Fertilizer Best Management Practices. Brussels. Belgium. March 7-9

Espinosa, J., \& García, J. P. 2010. Herramientas para mejorar la eficiencia de uso de nutrientes en maíz. Disponible en: http://nla.ipni.net/ipniweb/region/nla.nsf/e0f085ed5f091b1b852579000057902e/40ad1 eee26c802f00 5257a5300510c6d/\$FILE/ATT0EY3E.pdf/Herramientasmaiz.pdf

Espinosa, J., \& Mite, F. 2008. Búsqueda de eficiencia en el uso de nutrientes en Banano. Disponible en:https://www.researchgate.net/publication/242519031_BUSQUEDA_DE_EFICIENCIA_EN_EL_ USO_DE_NUTRIENTES_EN_BANANO

Espinosa, J., \& Mite, F. 2002. Estado actual y futuro de la nutrición y fertilización del banano. Revista Informaciones Agronómicas, 48, 4-9

Espinosa, J., \& Mite, F. 1992. Estado actual y futuro de la nutrición y fertilización del banano. Informaciones agronómicas

Fixen, P. 2010. Eficiencia de uso de nutrientes en el contexto de agricultura sostenible. Revista Informaciones Agronómicas, 76, 1-9

Fixen, P.E. 2005. Understanding and improving nutrients use efficiency as an application of information technology. Proceedings of the Symposium on Information Technology in Soils Fertility and Fertilizer Management, a satellite symposium at the XV International Plant Nutrition Colloquium, Beijin. China. Sep. 14-16

Furcal, P., \& Barquero, A. 2014. Fertilización del plátano con nitrógeno y potasio durante el primer ciclo productivo. Agronomía Mesoamericana, 25(2), 267-278

Furcal, P., \& Barquero, A. 2013. Respuesta del plátano a la fertilización con P, K y S durante el primer ciclo productivo. Agronomía Mesoamericana, 24(2), 317-327

García, F. O., \& Salvagiotti, F. 2015. Eficiencia de uso de nutrientes en sistemas agrícolas del cono sur de Latinoamérica. In XVIII Congreso Latinoamericano de la Ciencia del Suelo 
Guerrero, M. 2010. Guía técnica del cultivo del plátano. Programa MAG-CENTA-Frutales. CENTA (Centro Nacional de Tecnología Agropecuaria y Forestal Enrique Álvarez Córdova), El Salvador. Disponible en: http://www.centa.gob.sv/docs/guias/frutales/GUIA\%20CULTIVO\%20PLATANO\%202011.pdf

INEC. 2016. Encuesta de Superficie y Producción Agropecuaria Continua. Disponible en: http://www.ecuadorencifras.gob.ec/documentos/web-inec/Estadisticas_agropecuarias/espac/espac2016/Presentacion\%20ESPAC\%202016.pdf

Langdom PW, Whiley AW, Mayer RJ, Pegg KG, Smith MK 2008. The influence of planting density on the production of 'Goldfinger' (Musa spp., AAAB) in the subtropics. Scientia Horticulturae $115: 238-243$

López, O.R. 2002. Manual de producción de plátano basado en la experiencia de Zamorano. Tesis de Lic., Zamorano, Honduras. Disponible http://bdigital.zamorano.edu/bitstream/11036/2374/1/CPA-2002-T071.pdf

Palencia, G.E., S.R. Gómez, y J.E. Martín S. 2006. Manejo sostenible del cultivo de plátano. CORPOICA (Corporación Colombiana de Investigación Agropecuaria), Colombia

Parménides, F.-B. \& Barquero-Badilla, A., 2014. Fertilización del plátano con Nitrógeno y Potasio. Agron. Mesoam, 25(2), pp.267-278

SINAGAP. 2015. Boletín Situacional Plátano. Disponible en: http://sinagap.agricultura.gob.ec/phocadownloadpap/cultivo/2016/boletin_situacional_platano_2015 .pdf

Snyder, C. S., Bruulsema, T. W., \& IPNI. 2007. Nutrient use efficiency and effectiveness in North América: Indices of agronomic and environmental benefit. International Plant Nutrition Institute 